\title{
Two-photon absorption in a two-level system enabled by noise
}

\author{
V. V. Mkhitaryan ${ }^{1}$, C. Boehme ${ }^{1}$, J. M. Lupton ${ }^{1,2}$, and M. E. Raikh ${ }^{1}$ \\ 1 Department of Physics and Astronomy, University of Utah, Salt Lake City, UT 84112 \\ 2 Institut für Experimentelle und Angewandte Physik, \\ Universität Regensburg, Universitätsstr. 31, 93053 Regensburg, Germany
}

\begin{abstract}
We address the textbook problem of dynamics of a spin placed in a dc magnetic field and subjected to an ac drive. If the drive is polarized in the plane perpendicular to the dc field, the drive photons are resonantly absorbed when the spacing between the Zeeman levels is close to the photon energy. This is the only resonance when the drive is circularly polarized. For linearly polarized drive, additional resonances corresponding to absorption of three, five, and multiple odd numbers of photons is possible. Interaction with the environment causes the broadening of the absorption lines. We demonstrate that the interaction with environment enables the forbidden two-photon absorption. We adopt a model of the environment in the form of random telegraph noise produced by a single fluctuator. As a result of the synchronous time fluctuations of different components of the random field, the shape of the two-photon absorption line is non-Lorentzian and depends dramatically on the drive amplitude. This shape is a monotonic curve at strong drive, while, at weak drive, it develops a two-peak structure reminiscent of an induced transparency on resonance.
\end{abstract}

\section{INTRODUCTION}

It has been known for a long time $e^{\underline{1}-\underline{3}}$ that a spin placed in a magnetic field, $\boldsymbol{B}=\mathbf{z}_{0} B_{0}$, and subjected to a linear ac drive, $\boldsymbol{B}_{1}=\mathbf{x}_{0} B_{1} \cos \omega t$, exhibits Rabi oscillations when the driving frequency, $\omega$, is close to

$$
\omega_{2 p+1}=\frac{B_{0}}{2 p+1},
$$

where $p$ is integer. The frequency of $(2 p+1)$-photon Rabi oscillations is, within a factor, given by ${ }^{2} \frac{B_{1}^{2 p+1}}{B_{0}^{2 p}}$. Coupling to the environment ${ }^{4}$ causes the decay of the Rabi oscillations, i.e. dephasing. In the frequency domain, two split Rabi $\delta$-peaks in the absorption spectrum acquire a finite width and, upon further increasing of coupling to the environment, gradually merge into a single peak.

For some time now $\stackrel{5-13}{\underline{-13}}$ Rabi oscillations can be realized on individual two-level systems rather than on ensembles. In these realizations, the role of $\uparrow$ and $\downarrow$ states of a spin is played by different charge- or flux-states of a superconducting qubit.

Absence of even-photon absorption peaks follows from the Floquet description of the driven two-level systems. The argument presented in Ref. 1 is general and goes as follows.

The system relating the amplitudes of $\uparrow$ and $\downarrow$ projections of spin reads

$$
\begin{aligned}
& i \dot{C}_{\frac{1}{2}}=\frac{B_{0}}{2} C_{\frac{1}{2}}+B_{1} \cos \omega t C_{-\frac{1}{2}}, \\
& i \dot{C}_{-\frac{1}{2}}=-\frac{B_{0}}{2} C_{-\frac{1}{2}}+B_{1} \cos \omega t C_{\frac{1}{2}} .
\end{aligned}
$$

Searching for solutions in the form

$$
C_{\frac{1}{2}}=e^{i \lambda t} \sum_{-\infty}^{\infty} \alpha_{n} e^{i n \omega t}, C_{-\frac{1}{2}}=e^{i \lambda t} \sum_{-\infty}^{\infty} \beta_{n} e^{i n \omega t}
$$

where $\lambda$ is the Floquet exponent, reduces the system Eq. (28) to the infinite set of coupled equations

$$
\begin{aligned}
& {\left[(\lambda+n \omega)-\frac{B_{0}}{2}\right] \alpha_{n}=\frac{B_{1}}{2}\left(\beta_{n-1}+\beta_{n+1}\right),} \\
& {\left[(\lambda+n \omega)+\frac{B_{0}}{2}\right] \beta_{n}=\frac{B_{1}}{2}\left(\alpha_{n-1}+\alpha_{n+1}\right) .}
\end{aligned}
$$

In the limit of a weak drive, $B_{1} \ll B_{0}$, a single-photon resonance corresponds to $\lambda \approx-\frac{\omega}{2}$. For this $\lambda$ the brackets in front of $\alpha_{1}$ and $\beta_{0}$ become small. To find the value of $\lambda$ with accuracy up to the Bloch-Siegert shift, it is sufficient to truncate the system neglecting all $\alpha_{n}$ and $\beta_{n}$ except for $\alpha_{1}$ and $\beta_{0}$. We then obtain $\lambda= \pm \frac{1}{2}\left[\left(\omega-B_{0}\right)^{2}+B_{1}^{2}\right]^{1 / 2}$, which is the Rabi result.

A two-photon resonance, if it were allowed, would correspond to $\lambda \ll B_{0}$ so that the brackets in front of $\alpha_{1}$ and $\beta_{-1}$ become small. However, it is seen from the system Eq. (4) that $\alpha_{1}$ and $\beta_{-1}$ are completely decoupled. Indeed, $\alpha_{n}$ with $n$ odd is coupled only to $\beta_{n}$ with $n$ even and vice versa.

The message of the present paper is that for a drive frequency, $\omega$, close to $\frac{B_{0}}{2}$, coupling to the environment enables the resonant two-photon absorption. We calculate the shape of the absorption peak as a function of the drive amplitude and the parameters of the environment.

To illustrate the effect, we choose the simplest model of the environment illustrated in Fig. 1. It represents a single fluctuator with a telegraph-noise dynamics and coupled to the spin by the dipole-dipole interaction. We also demonstrate that for a general form of the environment, the magnitude of the two-photon absorption depends crucially on whether different components of the random magnetic fields are correlated or not.

We propose that this unexpected two-photon absorption, enabled by ambient noise, should be observable in devices made of organic semiconductors such as organic light-emitting diodes (OLEDs). 


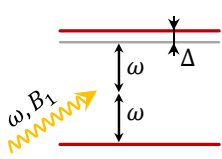

(a)

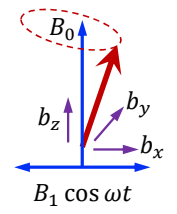

(b)

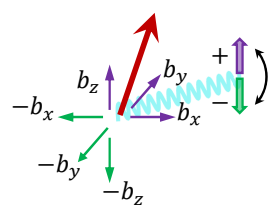

(c)
FIG. 1: (Color online) (a) Schematic illustration of the resonant two-photon absorption. The frequency, $\omega$, of drive with amplitude $B_{1} \ll \omega$ is tuned to the condition $\Delta=$ $\left(B_{0}-2 \omega\right) \ll \omega$, where $\Delta$ is the detuning; (b) For polarization of drive, $B_{1} \cos \omega t$, in the plane perpendicular to the field, $B_{0}$, two-photon absorption is forbidden. The environment can be viewed as a random magnetic field $\mathbf{b}(t)=\left(b_{x}(t), b_{y}(t), b_{z}(t)\right)$ fluctuating with time. Coupling of the two-level system to the environment enables the two-photon absorption; (c) We assume that the random field, $\mathbf{b}(t)$, is produced by a fluctuator coupled to the two-level system via the dipole-dipole interaction. Thus, the vector $\mathbf{b}(t)$ assumes two positions, $\pm\left(b_{x}, b_{y}, b_{z}\right)$, and switches between them at random moments. As a consequence, the components of $\mathbf{b}(t)$ fluctuate in-phase.

\section{EFFECTIVE HAMILTONIAN}

Consider a single fluctuator shown in Fig. 1, By virtue of the dipole-dipole interaction, this fluctuator produces a random magnetic field $\mathbf{b}(t)=\left(b_{x}(t), b_{y}(t), b_{z}(t)\right)$ at the location of the spin, see Fig. [1] As a result, the terms $\pm \frac{B_{0}}{2}$ in Eq. (28) become $\pm \frac{B_{0}+b_{z}(t)}{2}$, while the term $B_{1} \cos \omega t$ becomes $B_{1} \cos \omega t+b_{x}(t) \pm i b_{y}(t)$.

In order to incorporate the environment into the Floquet description, one has to assume that, instead of the common factor $e^{i \lambda t}$, the coefficients $\alpha_{n}$ and $\beta_{n}$ are themselves time-dependent. This generates the terms $\dot{\alpha}_{n}$ and $\dot{\beta}_{n}$ on the left-hand sides of the system Eq. (4). In fact, only the derivatives $\dot{\alpha}_{1}$ and $\dot{\beta}_{-1}$ should be kept. This is because we assume that the dynamics of the environment is slow, i.e. $\omega \tau \gg 1$, where $\tau$ is the characteristic correlation time of the environment. Under this condition, all the terms $\dot{\alpha}_{n}$ and $\dot{\beta}_{n}$ other than $\dot{\alpha}_{1}, \dot{\beta}_{-1}$ are added to much bigger terms $\left(n \omega+\frac{B_{0}}{2}\right) \alpha_{n}$ and $\left(n \omega-\frac{B_{0}}{2}\right) \beta_{n}$, respectively.

Coupling of the amplitudes $\alpha_{1}$ and $\beta_{-1}$ is mediated by the intermediate states with amplitudes $\alpha_{0}, \alpha_{-1}$ and $\beta_{0}$, $\beta_{1}$. Keeping only these six amplitudes, we arrive at the following truncated system

$$
\begin{aligned}
& i \dot{\alpha}_{1}-\frac{\Delta+b_{z}}{2} \alpha_{1}=\frac{B_{1}}{2} \beta_{0}+\frac{b_{-}}{2} \beta_{1}, \\
& -\frac{B_{0}}{2} \alpha_{0}=\frac{B_{1}}{2}\left(\beta_{-1}+\beta_{1}\right)+\frac{b_{-}}{2} \beta_{0}, \\
& \frac{B_{0}}{2} \beta_{0}=\frac{B_{1}}{2}\left(\alpha_{-1}+\alpha_{1}\right)+\frac{b_{+}}{2} \alpha_{0}, \\
& -B_{0} \alpha_{-1}=\frac{B_{1}}{2} \beta_{0}+\frac{b_{-}}{2} \beta_{-1}, \\
& B_{0} \beta_{1}=\frac{B_{1}}{2} \alpha_{0}+\frac{b_{+}}{2} \alpha_{1},
\end{aligned}
$$

$$
i \dot{\beta}_{-1}+\frac{\Delta+b_{z}}{2} \beta_{-1}=\frac{B_{1}}{2} \alpha_{0}+\frac{b_{+}}{2} \alpha_{-1} .
$$

where $b_{ \pm}=b_{x} \pm i b_{y}$ and

$$
\Delta=B_{0}-2 \omega
$$

is the detuning from the two-photon resonance. In the coefficients in front of the non-resonant amplitudes we have set $\omega=\frac{B_{0}}{2}$.

The strategy to analyze the system Eq. (6) is to express the intermediate amplitudes, $\alpha_{0}, \alpha_{-1}, \beta_{0}$, and $\beta_{1}$ in terms of $\alpha_{1}$ and $\beta_{-1}$. As a first step, we express $\alpha_{-1}$ and $\beta_{1}$ from the fourth and fifth equations and substitute them into the second and third equations. This yields

$$
\begin{aligned}
& -\left(B_{0}+\frac{B_{1}^{2}}{2 B_{0}}\right) \alpha_{0}-b_{-} \beta_{0}=B_{1}\left(\frac{b_{+}}{2 B_{0}} \alpha_{1}+\beta_{-1}\right), \\
& \left(B_{0}+\frac{B_{1}^{2}}{2 B_{0}}\right) \beta_{0}-b_{+} \alpha_{0}=B_{1}\left(\alpha_{1}-\frac{b_{-}}{2 B_{0}} \beta_{-1}\right) .
\end{aligned}
$$

Clearly, the term $\frac{B_{1}^{2}}{2 B_{0}}$ is much smaller than $B_{0}$ and can be neglected. This term contributes to the Bloch-Siegert shift of the two-photon resonance. Solving the system Eq. (8) in the leading order, we find

$$
\begin{aligned}
& \alpha_{0}=-\frac{B_{1}}{B_{0}}\left(\frac{b_{+}+2 b_{-}}{2 B_{0}} \alpha_{1}+\beta_{-1}\right), \\
& \beta_{0}=-\frac{B_{1}}{B_{0}}\left(\frac{2 b_{+}+b_{-}}{2 B_{0}} \beta_{-1}-\alpha_{1}\right) .
\end{aligned}
$$

Next, we express $\beta_{1}$ and $\alpha_{-1}$ using Eq. (9) and substitute them into the right-hand sides of the first and sixth equations of the system Eq. (6). Upon doing so, we conclude that the equations for $\alpha_{1}$ and $\beta_{-1}$ reduce to the pair of the Schrödinger equations generated by the effective Hamiltonian

$$
\hat{H}_{e f f}=\left(\Delta+b_{z}(t)\right) S_{z}+\tilde{b}_{x}(t) S_{x}
$$

where the effective field $\tilde{b}_{x}(t)$ is defined as

$$
\tilde{b}_{x}(t)=\frac{2 B_{1}^{2}}{B_{0}^{2}} b_{x}(t) .
$$

Note that the $b_{y}$-component of the random field drops out of the effective Hamiltonian. Technically, this happens because $\hat{H}_{e f f}$ contains the combination $b_{+}+b_{-}=2 b_{x}(t)$.

\section{ABSORPTION SPECTRUM}

\section{A. General expression}

In calculating the absorption coefficient from the Hamiltonian Eq. (10) we take advantage of the fact that $\tilde{b}_{x}$ is small, namely $\tilde{b}_{x} \tau \ll 1$. This justifies using the cumulant expansion ${ }^{14}$ to describe the dynamics of the 
disorder-averaged spin. In particular, the spin-spin correlation function, $\overline{S_{z}(0) S_{z}(t)}$, has the form

$$
\begin{aligned}
\overline{S_{z}(0) S_{z}(t)} & =\exp \left[-\int_{0}^{t} d t_{1} \int_{0}^{t_{1}} d t_{2} K\left(t_{1}-t_{2}\right)\right] \\
& =\exp \left[-\int_{0}^{t} d t_{1}\left(t-t_{1}\right) K\left(t_{1}\right)\right],
\end{aligned}
$$

where the correlator, $K(T)$, is defined as

$$
K(T)=\left\langle\tilde{b}_{x}(0) \tilde{b}_{x}(T) \cos \left[\Delta T+\int_{0}^{T} d t^{\prime} b_{z}\left(t^{\prime}\right)\right]\right\rangle .
$$

and $\langle\ldots\rangle$ stands for the averaging over the realizations of random time-dependent magnetic fields. Analytical form of the correlator is found in the Appendix within the model of the fluctuator, for which random magnetic fields $b_{z}(t)$ and $b_{x}(t)$ fluctuate in-phase and assume two values $\pm b_{x}$ and $\pm b_{z}$. The durations of intervals between switching events from $b_{x}, b_{z}$ to $-b_{x},-b_{z}$ are distributed following the Poisson distribution

$$
p\left(t_{i}\right)=\frac{1}{\tau} \exp \left(-\frac{t_{i}}{\tau}\right)
$$

where $\tau$ is the average time between the successive switchings.

It is convenient to cast the result obtained in the Appendix in the form

$$
K(T)=\frac{\tilde{b}_{x}^{2} \tau \cos (\Delta T)}{\left(1-b_{z}^{2} \tau^{2}\right)^{1 / 2}}\left[\frac{1}{\tau_{f}} \exp \left(-\frac{T}{\tau_{f}}\right)-\frac{1}{\tau_{s}} \exp \left(-\frac{T}{\tau_{s}}\right)\right]
$$

where $\tau_{f}$ and $\tau_{s}$ denote the fast and slow relaxation times defined as

$$
\tau_{f}=\frac{\tau}{1+\left(1-b_{z}^{2} \tau^{2}\right)^{1 / 2}}, \quad \tau_{s}=\frac{\tau}{1-\left(1-b_{z}^{2} \tau^{2}\right)^{1 / 2}} .
$$

It should be emphasized that Eq. (15) is derived for $b_{x}(t)$, $b_{z}(t)$ fluctuating in-phase. A dramatic consequence of these in-phase fluctuations is the sign minus between the two exponential terms. As a result, $K(T)$ changes sign as a function of $T$. If only $b_{x}(t)$ fluctuated, as is the case considered in Refs. 13, 15, 16, the two terms in Eq. (15) would add rather than subtract. Below, we will see that this difference has a dramatic effect on the absorption lineshape

$$
I(\Delta)=2 \int_{0}^{\infty} d t \cos (\Delta t) \overline{S_{z}(0) S_{z}(t)}
$$

using the spin-spin correlation function Eq. (12).

\section{B. Shape of the absorption spectrum}

Technically, the calculation is performed with the help of the relation

$$
\begin{aligned}
& \int_{0}^{t} d t_{1}\left(t-t_{1}\right) \cos \left(\Delta t_{1}\right) \exp \left(-\frac{t_{1}}{\tau_{0}}\right) \\
& =\frac{t \tau_{0}}{1+\Delta^{2} \tau_{0}^{2}}-\frac{\tau_{0}^{2}\left(1-\Delta^{2} \tau_{0}^{2}\right)}{\left(1+\Delta^{2} \tau_{0}^{2}\right)^{2}} \\
& +\tau_{0}^{2}\left[\frac{\left(1-\Delta^{2} \tau_{0}^{2}\right) \cos \Delta t-2 \Delta \tau_{0} \sin \Delta t}{\left(1+\Delta^{2} \tau_{0}^{2}\right)^{2}}\right] \exp \left(-\frac{t}{\tau_{0}}\right) .
\end{aligned}
$$

At long times, the term linear in $t$ dominates the result. With correlator Eq. (15), this term assumes the form

$$
\begin{aligned}
& \int_{0}^{t} d t_{1}\left(t-t_{1}\right) K\left(t_{1}\right) \\
& =\frac{\tilde{b}_{x}^{2} \tau t}{\left(1-b_{z}^{2} \tau^{2}\right)^{1 / 2}}\left[\frac{1}{1+\Delta^{2} \tau_{f}^{2}}-\frac{1}{1+\Delta^{2} \tau_{s}^{2}}\right] .
\end{aligned}
$$

Note that, for $\Delta=0$, the linear term vanishes. This is the consequence of the in-phase fluctuations of $b_{x}(t)$ and $b_{z}(t)$.

Further calculations are performed for the most interesting regime of the two-photon absorption, the "spectral narrowing" regime, ${ }^{17-19} b_{z} \tau \ll 1$. Under these conditions, the fast and slow times differ strongly, namely, $\tau_{s} \gg \tau_{f}$. Indeed, expanding Eq. (16) we get

$$
\tau_{f}=\frac{\tau}{2}, \quad \tau_{s}=\frac{2}{b_{z}^{2} \tau}
$$

At zero detuning, the exponent in Eq. (12) is dominated by subleading terms in Eq. (18). Thus, instead of Eq. (19) one has

$$
\begin{aligned}
& \left.\int_{0}^{t} d t_{1}\left(t-t_{1}\right) K\left(t_{1}\right)\right|_{\Delta=0} \\
& \approx \tilde{b}_{x}^{2} \tau\left[\tau_{s}\left(1-e^{-\frac{t}{\tau_{s}}}\right)-\tau_{f}\left(1-e^{-\frac{t}{\tau_{f}}}\right)\right] .
\end{aligned}
$$

Since $\tau_{s} \gg \tau_{f}$, the second term can be dropped. It then follows from Eq. (21) that the magnitude of absorption is governed by the dimensionless parameter

$$
\beta=\tilde{b}_{x}^{2} \tau \tau_{s}=2 \frac{\tilde{b}_{x}^{2}}{b_{z}^{2}}
$$

so that for $\beta \sim 1$, the characteristic time in the integral Eq. (17) is $t \sim \tau_{s}$. It also follows from Eq. (19) that for $\beta \sim 1$ the characteristic detuning is $\Delta \sim \tau_{s}^{-1}$. Indeed, in terms of parameter $\beta$, the leading term Eq. (19) can be cast in the form 

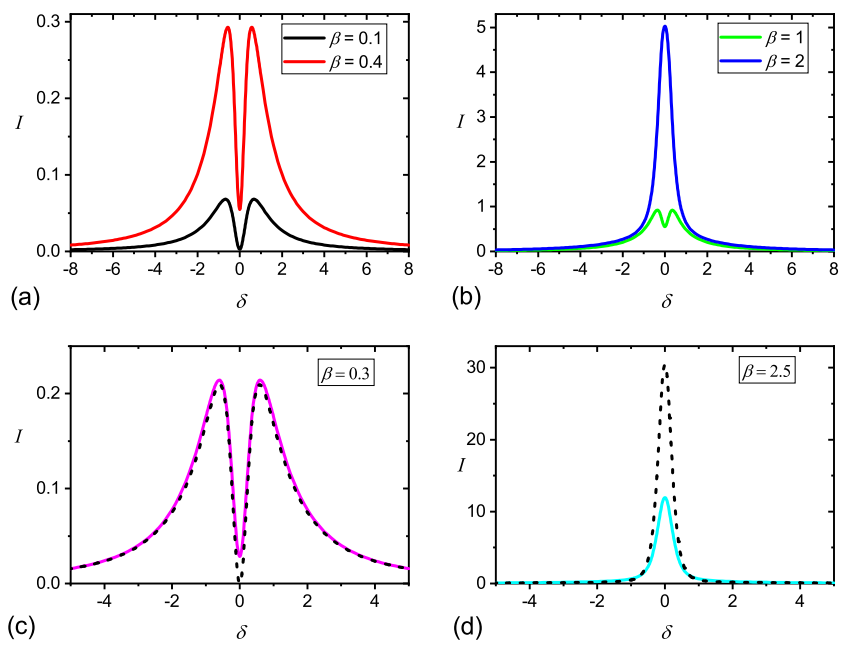

FIG. 2: (Color online) The shapes of the two-photon absorption spectra calculated numerically from Eq. (25) are plotted for small (a) and large (b) values of the parameter $\beta$ [Eq. (22)]. For low beta values, a dip appears around zero detuning. Upon the increase of $\beta \propto B_{1}^{4}$ the shape evolves from a two-peak structure to a single peak. Note that the widths of the curves are only weakly dependent on $\beta$. This dependency should be contrasted to the situation when only the $b_{x}$ component fluctuates. In this situation, the width grows linearly with $\beta$. In panels (c) and (d), the numerical curves are shown together with the approximate expressions Eq. (27) and Eq. (26), respectively (dotted lines). It is seen that Eq. (27) describes the small- $\beta$ behavior fairly accurately, while Eq. (26) captures the shape of the spectrum at large $\beta$.

$$
\frac{\beta \Delta^{2} \tau_{s} t}{1+\Delta^{2} \tau_{s}^{2}}
$$

It is now convenient to rewrite the expression Eq. (17) for the absorption spectrum by introducing the dimensionless variables

$$
t_{1}=\frac{t}{\tau_{s}}, \quad \delta=\Delta \tau_{s}
$$

In terms of these variables, Eq. (17) assumes the form

$$
\begin{aligned}
& I(\delta)=2 \tau_{s} \int_{0}^{\infty} d t_{1} \cos \delta t_{1} \exp \left(-\frac{\beta \delta^{2} t_{1}}{1+\delta^{2}}\right) \\
& \exp \left\{\beta \frac{\left(1-\delta^{2}\right)\left(1-e^{-t_{1}} \cos \delta t_{1}\right)+2 e^{-t_{1}} \delta \sin \delta t_{1}}{\left(1+\delta^{2}\right)^{2}}\right\} .
\end{aligned}
$$

Numerical plots of $I(\delta)$ for different $\beta$ are shown in Fig. 2. Our prime observation is that, for strong drive, $I(\delta)$ is a monotonic curve, while for a weak drive it develops a minimum at $\delta=0$. We have traced this anomalous behavior to the fact that the term $\cos \Delta t$ is present both as the prefactor of Eq. (24) and in the exponent. We emphasize again that oscillations in the exponent play a role only because the leading term Eq. (23) is suppressed at small $\delta$, which, in turn, is the consequence of the inphase fluctuations of $b_{x}(t)$ and $b_{z}(t)$. Concerning the width of the curves, they narrow down upon increasing drive. Although it is impossible to evaluate the integral Eq. (24) analytically, we were able to capture the behavior of numerical curves under certain assumptions. If we neglect the oscillating terms in the exponent completely, then the integral can be easily evaluated yielding

$$
I(\delta)=2 \tau_{s} \beta \frac{1+\delta^{2}}{\left(1+\delta^{2}\right)^{2}+\beta^{2} \delta^{2}} \exp \left\{\beta \frac{1-\delta^{2}}{\left(1+\delta^{2}\right)^{2}}\right\} .
$$

Not surprisingly, the above expression is a monotonic curve. For $\beta \ll 1$, the shape, $I(\delta)$, is a Lorentzian: $I(\delta) \propto \frac{1}{1+\delta^{2}}$, while for large $\beta$, it is again a Lorentzian $I(\delta) \propto \frac{1}{1+\beta^{2} \delta^{2}}$, but with a much smaller width.

In order to capture synchronous oscillations of the prefactor and the exponent, we perform the integration by parts in Eq. (24) and, subsequently, again neglect the oscillations in the exponent. The result again reduces to the elementary integrals and has the form

$$
\begin{aligned}
& I(\delta)=\tau_{s} \beta\left[\frac{2\left(1+\delta^{2}\right)}{\left(1+\delta^{2}\right)^{2}+\beta^{2} \delta^{2}}+\frac{1}{1+\delta^{2}(1+\beta)}\right. \\
& \left.-\frac{3\left(1+\delta^{2}\right)+\beta \delta^{2}}{4 \delta^{2}\left(1+\delta^{2}\right)^{2}+\left(1+\delta^{2}(1+\beta)\right)^{2}}\right] \exp \left\{\beta \frac{1-\delta^{2}}{\left(1+\delta^{2}\right)^{2}}\right\}
\end{aligned}
$$

The first term in Eq. (27) reproduces the previous result Eq. (26). On the other hand, for $\Delta=0$, Eq. (27) yields zero. From Fig. 2 we see that Eq. (26) describes the numerical results at large $\beta$ (i.e. under the conditions of strong drive), while Eq. (27) captures a minimum at weak drive. In other words, Eq. (26) completely neglects the synchronous oscillations, while Eq. (27) overestimates them. Roughly, one can argue that the true absorption spectrum lies in between the analytical predictions Eq. (26) and Eq. (27). Recalling that the parameter $\beta$ is proportional to the fourth power of drive implies that the shape of the absorption spectrum is extremely sensitive to the drive magnitude.

\section{Domain of applicability of the analytical results}

The form of the expressions Eqs. (26), (27) suggests that they do not apply in the domain $\beta \gg 1$. Indeed, exponential growth of the absorption with $\beta$ is unphysical. This prompts us to examine the validity of the general expression Eq. (12) for the spin-spin correlator. We will see that the exponential growth is the consequence of the in-phase fluctuations of $b_{x}(t)$ and $b_{z}(t)$. For uncorrelated $b_{x}(t), b_{z}(t)$ there is no unphysical growth and the criterion $\tilde{b}_{x} \tau \ll 1$ is sufficient to express the correlator in terms of the second cumulant. 
The easiest way to derive Eq. (12) is to analyze the system of equations of motion for the spin projections which follow from the effective Hamiltonian Eq. (10)

$$
\begin{aligned}
& \frac{\partial S_{x}}{\partial t}=-\left(\Delta+b_{z}(t)\right) S_{y}, \\
& \frac{\partial S_{y}}{\partial t}=-\tilde{b}_{x}(t) S_{z}+\left(\Delta+b_{z}(t)\right) S_{x}, \\
& \frac{\partial S_{z}}{\partial t}=\tilde{b}_{x}(t) S_{y} .
\end{aligned}
$$

Upon eliminating $S_{x}$ and $S_{y}$ and using the initial conditions $S_{x}(0)=S_{y}(0)=0$, we arrive to the following closed integral equation for $S_{z}(t)$

$$
\begin{aligned}
& S_{z}(t)=1 \\
& -\int_{0}^{t} d t_{1} \int_{0}^{t_{1}} d t_{2} \tilde{b}_{x}\left(t_{2}\right) \tilde{b}_{x}\left(t_{1}\right) \cos \left[\Delta t_{1}+\int_{t_{2}}^{t_{1}} d t^{\prime} b_{z}\left(t^{\prime}\right)\right] S_{z}\left(t_{2}\right) .
\end{aligned}
$$

This equation is exact and applies for an arbitrary noise realization. It can be reduced to a closed integral equation for $\overline{S_{z}(t)}$, the average over the noise realizations. The form of the closed integral equation is the following 14

$$
\overline{S_{z}(t)}=1-\int_{0}^{t} d t_{1} \int_{0}^{t_{1}} d t_{2} K\left(t_{1}-t_{2}\right) \overline{S_{z}\left(t_{2}\right)},
$$

where $K(t)$ is the noise-averaged kernel in Eq. (29) given by Eq. (13). Importantly, the reduction of Eq. (29) to Eq. (30) is valid under the assumption that the decay of $\overline{S_{z}(t)}$ is much slower than the decay of $K(t)$. Solving Eq. (30) by successive iterations reproduces the result Eq. (12).

Now we have to check the basic assumption that $\overline{S_{z}(t)}$ falls off slower than $K(t)$. If only $b_{x}(t)$ fluctuated, i.e. $b_{z}(t)=0$, then the time-scale for the change of $K(t)$ would be $\tau$, [see Eq. [15)], while the time-scale for the decay of $\overline{S_{z}(t)}$ would be $\left(\tilde{b}_{x}^{2} \tau\right)^{-1}$. This yields the conventional condition $\tilde{b}_{x} \tau \ll 1$, which we assumed to be met.

For the in-phase fluctuations of $b_{x}(t)$ and $b_{z}(t)$, the correlator falls off over much longer time $\tau_{s} \gg \tau$. On the other hand, $\overline{S_{z}(t)}$ decays over time much longer than $\left(\tilde{b}_{x}^{2} \tau\right)^{-1}$. This decay time can be estimated from Eq. (19) to be $t \sim\left(\tilde{b}_{x}^{2} \tau\right)^{-1}\left(\frac{1+\Delta^{2} \tau_{s}^{2}}{\Delta^{2} \tau_{s}^{2}}\right)$. Then the basic assumption reduces to the inequality

$$
\beta<\frac{1+\delta^{2}}{\delta^{2}}
$$

This condition forbids large values of $\beta$, i.e. small values of $b_{z} \ll \tilde{b}_{x}$. In the opposite limit of small $b_{z}$ the absorption spectrum is Lorentizan with the width $\Delta=\tilde{b}_{x}^{2} \tau$, i.e. it grows with drive as $B_{1}^{4}$.

\section{DISCUSSION}

In this Section we discuss the experimental feasibility of the predicted effect and put it into a general perspective.

\section{A. General remarks}

1. The phenomenon of two-photon absorption is widely used in many different domains of science and engineering, primarily due to its non-linear dependency on intensity which opens the possibility of depth resolution in light-matter interaction, but it is usually considered in the context of electronic dipole transitions. Because of the angular momentum of the photon, the parity of an electronic state changes after absorption of a photon but remains the same under two-photon absorption. One-and two-photon absorption therefore probe different electronic states under electronic dipole transition. For magnetic dipole transitions, in contrast, because of the change in spin quantum number, the matrix element becomes non-vanishing between states of the same parity, irrespective of whether one or two photons are absorbed at once. Magnetic-dipole two-photon absorption therefore offers a method to excite odd-parity states, which are spin forbidden, and has been used to study para-excitons in semiconductors and insulators 20 .

2. While our result of the absence of absorption at zero detuning bears some superficial resemblance of conventional electromagnetically induced transparency (EIT), analogues of which can also be generated in magneticresonant systems, $\stackrel{3,21}{=}$ we stress that the origin is completely different. In EIT, quantum interference occurs between different transition pathways of a multilevel system, whereas here, we consider solely the effect of noise on two-level systems.

In fact, the origin of the dip at zero detuning is a direct consequence of the in-phase telegraph-noise fluctuations of $b_{x}(t)$ and $b_{z}(t)$. Indeed, upon setting $\Delta=0$ in the effective Hamiltonian Eq. (10), it assumes the form $\hat{H}_{\text {eff }}=b_{z}(t) S_{z}+\tilde{b}_{x} S_{x}$. Note that the eigenvalues of this Hamiltonian, $\pm\left[b_{z}(t)^{2}+\tilde{b}_{x}(t)^{2}\right]^{1 / 2}$, are timeindependent. As $b_{z}, \tilde{b}_{x}$ change to $-b_{z},-\tilde{b}_{x}$, two eigenvectors remain the same while their eigenvalues interchange. As a result, despite the randomness of the switching moments, $S_{z}(t)$ oscillates with time. Hence, there is no absorption.

\section{B. Relevance to organic semiconductors}

1. In addition to superconducting qubits $s^{-11,22}$, a natural setup to verify our predictions is nuclear magnetic resonance, see e.g. Ref. 23, although identification of individual spectral lines requires very high sensitivity and 
selectivity which can be hard to achieve given the limits imposed on sampling frequency by the small nuclear gyromagnetic ratio. On the other hand, the two-photon absorption predicted above is proportional to the fourth power of the ratio $B_{1} / B_{0}$, and is generally very weak. Spin resonance in electronic systems appears to offer a more promising avenue. Indeed, a very high sensitivity of optical and electrical detection of magnetic resonances has recently been demonstrated in organic semiconductors, see Refs. 24 28. Crucially, instead of measuring magnetic polarization, these experiments report on the permutation symmetry of pairs of spins, and are therefore much more sensitive than conventional measurements of magnetization. Also, organic semiconductors are appealing because of the long spin lifetimes of carriers $\underline{29}$

2. Experimental observation of the predicted effect necessitates strong drive conditions where the carrier-wave frequency $B_{0}$ is of order of the Rabi frequency $B_{1}$ and, in addition, both frequencies are comparable to that of the noise. Since $\mathrm{MHz}$ magnetic resonances are detectable electrically in OLEDs, $\stackrel{30}{\longrightarrow}$ such systems should offer an avenue for observing forbidden two-photon transitions.

3. In contrast to other well-defined spin systems such as color centers in diamond, spin-1/2 paramagnetic species in organic semiconductors do not experience any appreciable zero-field splitting. As a consequence, magnetic resonance can even occur at zero external field, mediated alone by the local hyperfine fields $\frac{30}{\underline{3}}$ Electrically detected magnetic resonance (EDMR) in OLEDs bears some resemblance to magnetic-field effects and magnetic resonance phenomena in radical-pair-based chemical reactions ${ }^{31.32}$ but offers more flexibility in terms of choosing the crucial ratio between carrier-wave frequency $\left(B_{0}\right)$, Rabi frequency $\left(B_{1}\right)$ and the noise amplitude, which is determined by the local hyperfine fields. These fields, in turn, can be tuned chemically by controlling the isotopic ratio of hydrogen and deuterium. ${ }^{33}$ OLEDs offer the crucial advantage in EDMR experiments that the active device area undergoing resonance can be shrunk in size almost arbitrarily. This control is crucial not only to enable the generation of high resonant driving field amplitudes, $\stackrel{34}{=}$ but also to ensure a high level of homogeneity of the $B_{0}$ field. The latter aspect is crucial in controlling the degree of static disorder of the Zeeman-split two-level system with respect to the magnitude of dynamic disorder, which enables the dipoleforbidden two-photon transition.

4. Since an OLED operates by spin-dependent recombination of electrons and holes, EDMR does not, strictly, probe a two-level system, but two effectively degenerate two-level systems of the Zeeman-split electron and hole spin levels. Under strong drive, when the drive amplitude exceeds the variation in the expectation value of the hyperfine fields, electron and hole resonance become indistinguishable so that a new Dicke-type "superradiant" spin- $1 / 2$ resonant species emerges $\stackrel{33}{ }$ Since spin-orbit coupling and resulting shifts of the gyromagnetic ratio are

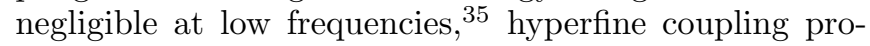

vides the only source of inhomogeneous broadening of the resonance. Recently, clear Zeeman resonances in EDMR were reported at frequencies as low as $5 \mathrm{MHz}$ for a hydrogenated organic semiconductor; $; \underline{30}$ for deuterated materials, this limit is expected to be much lower. It should therefore be straightforward to find a suitable parameter space where the resonance frequency is of order the typical noise frequency.

\section{CONCLUDING REMARKS}

1. In spirit, the noise-induced absorption bares some similarity to the hyperfine-induced excitation of the electron spin resonance predicted and observed in Ref. 37. In simple terms, the idea of Ref. 37 can be explained as follows. Suppose that the driving field, $B_{1}$, is absent, but the quantum dot confining an electron is "shaken" in space by an ac electric field with resonant frequency. As a result, hyperfine fields of nuclei inside the dot also change with time with resonant frequency and play the role of $B_{1}$. Since the hyperfine fields constitute the environment, the resonance observed in Ref. 37 can be viewed as environment-mediated.

In fact, the setup in experiment $\underline{37}$ included two quantum dots. Beating of electrons between the dots can be viewed as pseudospin dynamics. The shaking of the dots then couples the true electron spin dynamics to pseudospin dynamics $\frac{38}{2}$

2. Description of the ac absorption within the Bloch equations,$\stackrel{39}{=}$ where the bath is modeled by the spin-lattice relaxation time, $T_{1}$, does not capture the two-photon resonance. In order to replicate a number of nontrivial effects, a microscopic model of the environment must be specified. Moreover, it is necessary to go beyond the Bloch-Redfield description, as discussed in e.g. Ref. 40. For example, modeling of the environment by a periodic modulation of the applied field, can also allow twophoton absorption to occur ${ }^{23}$

3. While we have used the language of a spin driven by an ac magnetic field, the results apply quite generally for any driven two-level system. Usually, as, e.g., in Refs. 41 49, the effect of the environment on multiphoton absorption is studied under the conditions where this absorption is allowed even in the absence of environment. By contrast, our main message here is that the environment itself can actually enable an absorption which is otherwise forbidden.

4. Qualitative explanation of the absence of evenphoton resonances without an interaction with the bath is that linearly polarized drive has matrix elements $|\downarrow\rangle \rightarrow|\uparrow\rangle$ and $|\uparrow\rangle \rightarrow|\downarrow\rangle$, but not $|\downarrow\rangle \rightarrow|\downarrow\rangle$ or $|\uparrow\rangle \rightarrow|\uparrow\rangle$. Thus, each interaction with the drive is accompanied by a spin-flip. Even number of interactions with the drive necessarily returns the spin to the initial state, thus forbidding the absorption of the even number of drive quanta. As mentioned in Ref. 2 and elaborated on in Refs. 45, 47, a tilt of the dc field away from the 
plane normal to the drive polarization results in the matrix elements $|\downarrow\rangle \rightarrow|\downarrow\rangle$ and $|\uparrow\rangle \rightarrow|\uparrow\rangle$ becoming nonzero, thus allowing the absorption of an even number of drive quanta. In our study, the dc field is normal to the drive, yet the fluctuating field produced by the environment can be viewed as a time-dependent tilt of the de field.

5. A formal reason why in-phase fluctuations of $b_{x}(t)$ and $b_{z}(t)$ result in an unconventional shape of the twophoton absorption is the following. The argument of the cosine in the correlator Eq. (13) contains the integral $\int_{0}^{T} d t b_{z}(t)$, and the prefactor has the form $\tilde{b}_{x}(0) \tilde{b}_{x}(t)$. As a result of the in-phase fluctuations, $\tilde{b}_{x}(t)$ and $b_{z}(t)$ are proportional to each other. Thus, the prefactor is proportional to the derivative of the argument of the cosine, so that the correlator itself becomes proportional to the full time derivative of the oscillating function. The leading term Eq. (19) is proportional to the integral of the correlator. Thus, it is not surprising that this integral is zero for $\Delta=0$. We then conclude that for environment of a spin representing not a single but an ensemble of fluctuators, for which $b_{x}$ and $b_{z}$ are different but fluctuate in-phase, the above property still holds. Thus, we would intuitively expect the double-peaked shape of the absorption spectrum to emerge.

6. It is instructive to put our results into a more general perspective of spectral narrowing $\underline{17}-\underline{19}$. The randomness of $b_{z}$ emulates the spectral narrowing. However, if $b_{x}$ fluctuates in phase with $b_{z}$, the effect of spectral narrowing is undone. The same effect can be reformulated in the language of Franck-Condon physics. Within the Franck-Condon picture, the optical matrix elements are renormalized due to coupling of the electronic level to nuclear vibrations. If, hypothetically, the transition between electronic levels was due to the same vibrations, the Franck-Condon selection rule would be lifted. In this regard, note that breaking of the Franck-Condon principle occurs in multi-level systems such as molecules, where different electronic transitions intermix because of mutually shared vibrations and electronic and vibronic transitions are no longer independent $\underline{36}$

\section{ACKNOWLEDGEMENTS}

V. V. M. and M. E. R. were supported by the Department of Energy, Office of Basic Energy Sciences, Grant No. DE-FG02-06ER46313.

\section{APPENDIX}

From the definition Eq. (13) it is apparent that the $\Delta$-dependence of the correlator is $\cos \Delta T$. Thus, it is sufficient to carry out calculations for $\Delta=0$. For the telegraph noise, the correlation function, $K(T)$, can be expressed as a sum

$$
K(T)=\tilde{b}_{x}^{2} \sum_{n=0}^{\infty}(-1)^{n} \int_{0}^{\infty} \frac{d t_{1}}{\tau} e^{-\frac{t_{1}}{\tau}} \ldots \int_{0}^{\infty} \frac{d t_{n+1}}{\tau} e^{-\frac{t_{n+1}}{\tau}}
$$

$$
\times e^{i b_{z}\left(t_{1}-t_{2}+\cdots(-1)^{n} t_{n+1}\right)}\left[\theta\left(T-\sum_{k=1}^{n} t_{k}\right)-\theta\left(T-\sum_{k=1}^{n+1} t_{k}\right)\right] .
$$

Here, $\left\{t_{k}\right\}_{k=1}^{n+1}$ are the time moments at which $b_{z}(t)$ flips its sign. The factor $(-1)^{n}$ ensures that $b_{x}(t)$ flips the sign at the same time moments. The difference of $\theta$ functions in Eq. (32) guarantees that in the time interval $(0, T)$ the field $b_{z}$ changes its sign exactly $n$ times, so that $\sum_{k=1}^{n} t_{k}<T<\sum_{k=1}^{n+1} t_{k}$. Taking the integral over $t_{n+1}$ by parts leads to

$$
\begin{aligned}
& K(T)=\tilde{b}_{x}^{2} \sum_{n=0}^{\infty}(-1)^{n} \int_{0}^{\infty} \frac{d t_{1}}{\tau} \cdots \int_{0}^{\infty} \frac{d t_{n}}{\tau} \int_{0}^{\infty} d t_{n+1} \\
& \times e^{-\sum_{j=1}^{n+1} \frac{t_{j}}{\tau}} e^{i b_{z}\left(t_{1}-t_{2}+\cdots(-1)^{n} t_{n+1}\right)} \delta\left(T-\sum_{k=1}^{n+1} t_{k}\right) .
\end{aligned}
$$

Using the integral representation, $\delta(t)=\int_{-\infty}^{\infty} \frac{d s}{2 \pi} e^{i s t}$, in the integrand and taking the individual integrals yields

$$
K(T)=\tilde{b}_{x}^{2} \sum_{k=1}^{\infty} \int_{-\infty}^{\infty} \frac{d s}{2 \pi} e^{i s T} \frac{i\left(s+b_{z}\right) \tau^{2}}{\left[(1+i s \tau)^{2}+b_{z}^{2} \tau^{2}\right]^{k}}
$$

After taking the sum and symmetrizing with respect to $\pm b_{z}(0)$, we find

$$
K(T)=\tilde{b}_{x}^{2} \int_{-\infty}^{\infty} \frac{d s}{2 \pi} e^{i s T} \frac{i s \tau^{2}}{(1+i s \tau)^{2}+b_{z}^{2} \tau^{2}-1}
$$

The integral is calculated by finding the contributions of two poles at $s=\frac{i}{\tau} \pm\left(b_{z}^{2}-\frac{1}{\tau^{2}}\right)^{1 / 2}$. For $b_{z} \tau>1$ we find

$$
\begin{aligned}
& K(T)=\tilde{b}_{x}^{2} \exp \left(-\frac{T}{\tau}\right) \\
& \times\left\{\cos \left[\frac{T}{\tau}\left(b_{z}^{2} \tau^{2}-1\right)^{1 / 2}\right]-\frac{\sin \left[\frac{T}{\tau}\left(b_{z}^{2} \tau^{2}-1\right)^{1 / 2}\right]}{\left(b_{z}^{2} \tau^{2}-1\right)^{1 / 2}}\right\} .
\end{aligned}
$$

For $b_{z} \tau<1$ Eq. (35) yields

$$
\begin{aligned}
& K(T)=\tilde{b}_{x}^{2} \exp \left(-\frac{T}{\tau}\right) \\
& \times\left\{\cosh \left[\frac{T}{\tau}\left(1-b_{z}^{2} \tau^{2}\right)^{1 / 2}\right]-\frac{\sinh \left[\frac{T}{\tau}\left(1-b_{z}^{2} \tau^{2}\right)^{1 / 2}\right]}{\left(1-b_{z}^{2} \tau^{2}\right)^{1 / 2}}\right\} .
\end{aligned}
$$

We again emphasize that the factor $(-1)^{n}$ in the definition Eq. (32) had transformed into the signs minus in the final results Eqs. (36), (37) leading to a peculiar shape of the two-photon absorption. Physically, repeated changes of signs of $b_{x}$ between the same to values ${ }^{50}$ and in-phase with $b_{z}$ leads to averaging out of the effect of noise. 
1 S. H. Autler and C. H. Townes, "Stark Effect in Rapidly Varying Fields," Phys. Rev. 100, 703 (1955).

2 J. H. Shirley, "Solution of the Schrödinger equation with a hamiltonian periodic in time," Phys. Rev. 138, B979 (1965).

3 M. Kälin, M. Fedin, I. Gromov, and A. Schweiger, Multiple-photon transitions in EPR spectroscopy, in Novel NMR and EPR techniques, edited by J. Dolinšek, M. Vilfan, and S. Žumer, Lecture Notes in Physics, (Springer, Berlin, 2006).

4 M. Grifoni and P. Hänngi, Phys. Rep. 304, 229 (1998).

5 J. E. Mooij, T. P. Orlando, L. Levitov, L. Tian, C. H. van der Wal, and S. Lloyd, "Josephson persistent-current qubit," Science 285, 1036 (1999).

6 Y. Nakamura, Y. A. Pashkin, and J. S. Tsai, "Coherent control of macroscopic quantum states in a single-Cooperpair box," Nature (London) 398, 786 (1999).

7 C. H. van der Wal, A. C. J. ter Haar, F. K. Wilhelm, R. N. Schouten, C. J. P. M. Harmans, T. P. Orlando, S. Lloyd, and J. E. Mooij, "Quantum Superposition of Macroscopic Persistent-Current States," Science 290, 773 (2000).

8 D. Vion, A. Aassime, A. Cottet, P. Joyez, H. Pothier, C. Urbina, D. Esteve, and M. H. Devoret, "Superconducting tunable flux qubit with direct readout scheme," Science 296, 886 (2002).

9 I. Chiorescu, Y. Nakamura, C. J. P. M. Harmans, and J. E. Mooij, "Coherent Quantum Dynamics of a Superconducting Flux Qubit," Science 299, 1869 (2003).

10 E. Collin, G. Ithier, A. Aassime, P. Joyez, D.Vion, and D. Esteve, "NMR-like Control of a Quantum Bit Superconducting Circuit," Phys. Rev. Lett. 93, 157005 (2004).

11 Ya. S. Greenberg, E. Iljichev, and A. Izmalkov, "Lowfrequency Rabi spectroscopy for a dissipative two-level system," Europhys. Lett.72, 880 (2005).

12 S. N. Shevchenko, S. Ashhab, and F. Nori, "Landau-ZenerStückelberg interferometry," Phys. Rep. 492, 1 (2010).

13 E. Paladino, Y. M. Galperin, G. Falci, and B. L. Altshuler, " $1 / f$ noise: Implications for solid-state quantum information," Rev. Mod. Phys. 86, 361 (2014).

14 N. van Kampen, Stochastic Processes in Physics and Chemistry (North-Holland, 1981).

15 J. Bergli and L. Faoro, "Exact solution for the dynamical decoupling of a qubit with telegraph noise," Phys. Rev. B 75, 054515 (2007).

16 J. Bergli, Y. M. Galperin, and B. L. Altshuler, "Decoherence in qubits due to low-frequency noise," New J. Phys. 11, 025002 (2009).

17 P. W. Anderson and P. R. Weiss, "Exchange Narrowing in Paramagnetic Resonance," Rev. Mod. Phys. 25, 269 (1953).

18 R. Kubo, "Note on the Stochastic Theory of Resonance Absorption," J. Phys. Soc. Jpn. 9, 935 (1954).

19 J. R. Klauder and P. W. Anderson, "Spectral Diffusion Decay in Spin Resonance Experiments," Phys. Rev. 125, (1962).

20 D. Frohlich, M. Itoh, and C. Pahlkelerch, "2-photon spectroscopy of odd-parity states," Phys. Rev. Lett. 72, 1001 (1994).

21 M. Kälin, I. Gromov, and A. Schweiger, "Transparency in two-level spin systems induced by a longitudinal field," Phys. Rev. A 69, 033809 (2004).
22 A. Wallraff, T. Duty, A. Lukashenko, and A. V. Ustinov, "Multiphoton transitions between energy levels in a current-biased Josephson tunnel junction," Phys. Rev. Lett. 90, 037003 (2003).

23 R. Glenn, M. E. Limes, B. Pankovich, B. Saam, and M. E. Raikh, "Magnetic resonance in slowly modulated longitudinal field: Modified shape of the Rabi oscillations," Phys. Rev. B 87, 155128 (2013).

24 D. R. McCamey, K. J. van Schooten, W. J. Baker, S.-Y. Lee, S.-Y. Paik, J. M. Lupton, and C. Boehme, "HyperfineField-Mediated Spin Beating in Electrostatically Bound Charge Carrier Pairs," Phys. Rev. Lett. 104, 017601 (2010).

25 W. J. Baker, D. R. McCamey, K. J. van Schooten, J. M. Lupton, and C. Boehme, "Differentiation between polaronpair and triplet-exciton polaron spin-dependent mechanisms in organic light-emitting diodes by coherent spin beating," Phys. Rev. B 84, 165205 (2011).

26 W. J. Baker, K. Ambal, D. P. Waters, R. Baarda, H. Morishita, K. van Schooten, D. R. McCamey, J. M. Lupton, and C. Boehme, "Robust Absolute Magnetometry with Organic Thin-Film Devices," Nature Commun. 3, 898 (2012).

27 C. Boehme and J. M. Lupton, "Challenges for organic spintronics," Nat. Nanotechnology 8, 612 (2013).

28 S. L. Bayliss, N. C. Greenham, R. H. Friend, H. Bouchiat, and A. D. Chepelianskii, "Spin-dependent recombination probed through the dielectric polarizability," Nat. Commun. 6, 8534 (2015).

29 H. Malissa, M. Kavand, D. P. Waters, K. J. van Schooten, P. L. Burn, Z. V. Vardeny, B. Saam, J. M. Lupton, and C. Boehme, "Room-temperature coupling between electrical current and nuclear spins in OLEDs," Science 345, 1487 (2014).

30 T. Grünbaum, S. Milster, H. Kraus, W. Ratzke, S. Kurrmann, V. Zeller, S. Bange, C. Boehme, and J. M. Lupton, "OLEDs as models for bird magnetoception: detecting electron spin-resonance in geomagnetic fields," arXiv: 1906.03090

31 C. T. Rodgers, C. J. Wedge, S. A. Norman, P. Kukura, K. Nelson, N. Baker, K. Maeda, K. B. Henbest, P. J. Hore, and C. R. Timmel, "Radiofrequency polarization effects in zero-field electron paramagnetic resonance," Phys. Chem. Chem. Phys. 11, 6569 (2009).

32 C. J. Wedge, J. C. S. Lau, K.-A. Ferguson, S. A. Norman, P. J. Hore, and C. R. Timmel, "Spin-locking in lowfrequency reaction yield detected magnetic resonance," Phys. Chem. Chem. Phys. 15, 16043 (2013).

33 D. P. Waters, G. Joshi, M. Kavand, M. E. Limes, H. Malissa, P. L. Burn, J. M. Lupton, and C. Boehme, "The spin-Dicke effect in OLED magnetoresistance," Nature Phys. 11, 910 (2015).

34 S. Jamali, G. Joshi, H. Malissa, J. M. Lupton, and C. Boehme, "Monolithic OLED-Microwire Devices for Ultrastrong Magnetic Resonant Excitation," Nano Letters 17, 4648 (2017).

35 H. Malissa, R. Miller, D. L. Baird, S. Jamali, G. Joshi, M. Bursch, S. Grimme, J. van Tol, J. M. Lupton, and C. Boehme, "Revealing weak spin-orbit coupling effects on charge carriers in a pi-conjugated polymer," Phys. Rev. B 97, 161201(R) (2018). 
${ }^{36}$ P. Wilhelm, J. Vogelsang, N. Schönfelder, S. Höger, and J. M. Lupton, "Anomalous Linear Dichroism in Bent Chromophores of pi-conjugated Polymers: Departure from the Franck-Condon Principle," Phys. Rev. Lett. 122, 057402 (2019).

37 E. A. Laird, C. Barthel, E. I. Rashba, C. M. Marcus, M. P. Hanson, and A. C. Gossard, "Hyperfine-Mediated GateDriven Electron Spin Resonance," Phys. Rev. Lett. 99, 246601 (2007).

38 E. I. Rashba, "Mechanism of half-frequency electric dipole spin resonance in double quantum dots: Effect of nonlinear charge dynamics inside the singlet manifold," Phys. Rev. B 84, 241305(R) (2011).

39 F. Bloch, "Nuclear Induction", Phys. Rev. 70, 4604 (1946).

${ }^{40}$ V. V. Mkhitaryan and V. V. Dobrovitski, "Decay of the rotary echoes for the spin of a nitrogen-vacancy center in diamond," Phys. Rev. B 89, 224402 (2014).

41 A. P. Saiko, S. A. Markevich, and R. Fedaruk, "Multiphoton Raman transitions and Rabi oscillations in driven spin systems," Phys. Rev. A 98, 043814 (2018).

42 D. Gagnon, F. Fillion-Gourdeau, J. Dumont, C. Lefebvre, and S. MacLean, "Suppression of Multiphoton Resonances in Driven Quantum Systems via Pulse Shape Optimization," Phys. Rev. Lett. 119, 053203 (2017).

43 A. Settineri, V. Macrí, A. Ridolfo, O. D. Stefano, A. F. Kockum, F. Nori, and S. Savasta, "Dissipation and thermal noise in hybrid quantum systems in the ultrastrong- coupling regime," Phys. Rev. A 98, 053834 (2018).

44 M. C. Goorden, M. Thorwart, and M. Grifoni, "Entanglement Spectroscopy of a Driven Solid-State Qubit and Its Detector Phys. Rev. Lett. 93, 267005 (2004).

45 J. Hausinger and M. Grifoni, "Dissipative two-level system under strong ac driving: A combination of Floquet and Van Vleck perturbation theory," Phys. Rev. A 81, 022117 (2010).

46 Y. Yan, Z. Lü, J. Luo, and H. Zheng, "Effects of counterrotating couplings of the Rabi model with frequency modulation," Phys. Rev. A 96, 033802 (2017).

47 Y. Yan, Z. Lü, J. Luo, and H. Zheng, "Multiphotonresonance-induced fluorescence of a strongly driven twolevel system under frequency modulation," Phys. Rev. A 97, 033817 (2018).

48 J. Romhányi, G. Burkard, and A. Pályi, "Subharmonic transitions and Bloch-Siegert shift in electrically driven spin resonance," Phys. Rev. B 92, 054422 (2015).

49 J. V. Koski, A. J. Landig, A. Pályi, P. Scarlino, C. Reichl, W. Wegscheider, G. Burkard, A. Wallraff, K. Ensslin, and T. Ihn, "Floquet Spectroscopy of a Strongly Driven Quantum Dot Charge Qubit with a Microwave Resonator," Phys. Rev. Lett. 121, 043603 (2018).

50 R. C. Roundy and M. E. Raikh, "Tunnel magnetoresistance in organic spin valves in the regime of multistep tunneling," Phys. Rev. B 88, 205206 (2013). 